Katja Thomas, MD

Judith Eisele, MD

Francisco Alejandro

Rodriguez-Leal, MD

Undine Hainke, MD

Tjalf Ziemssen, MD

Correspondence to Prof. Dr. Tjalf Ziemssen: tjalf.ziemssen@uniklinikumdresden.de
Supplemental data at Neurology.org/nn

\section{Acute effects of alemtuzumab infusion in patients with active relapsing-remitting $\mathrm{MS}$ OPEN}

\section{ABSTRACT}

Objective: Alemtuzumab exerts its clinical efficacy by its specific pattern of depletion and repopulation of different immune cells. Beyond long-term immunologic and clinical data, little is known about acute changes in immunologic and routine laboratory parameters and their clinical relevance during the initial alemtuzumab infusion.

Methods: Fifteen patients with highly active MS were recruited. In addition to parameters including heart rate, blood pressure, body temperature, and monitoring of adverse events, complete blood cell count, liver enzymes, kidney function, acute-phase proteins, serum cytokine profile, complement activation, peripheral immune cell distribution, and their potential of cytokine release were investigated prior to and after methylprednisolone and after alemtuzumab on each day of alemtuzumab infusion.

Results: After the first alemtuzumab infusion, both the total leukocyte and granulocyte counts markedly increased, whereas lymphocyte counts dramatically decreased. In addition to lymphocyte depletion, cell subtypes important for innate immunity also decreased within the first week after alemtuzumab infusion. Although patients reported feeling well, C-reactive protein and procalcitonin peaked at serum levels consistent with septic conditions. Increases in liver enzymes were detected, although kidney function remained stable. Proinflammatory serum cytokine levels clearly rose after the first alemtuzumab infusion. Alemtuzumab led to impaired cytokine release ex vivo in nondepleted cells. Normal clinical parameters and mild adverse events were presented.

Conclusions: Dramatic immunologic effects were observed. Standardized infusion procedure and pretreatment management attenuated infusion-related reactions. Alemtuzumab-mediated effects led to artificially altered parameters in standard blood testing. We recommend clinical decision-making based on primarily clinical symptoms within the first alemtuzumab treatment week. Neurol Neuroimmunol Neuroinflamm 2016;3:e228; doi: 10.1212/NXI.0000000000000228

\section{GLOSSARY}

APC = antigen presenting cells; $\mathbf{C R P}=$ C-reactive protein; $\mathbf{D C}=$ dendritic cell; IAR $=$ infusion-associated reaction; IFN = interferon; IL = interleukin; LDH = lactate dehydrogenase; LPS = lipopolysaccharide; $\mathbf{M S}=$ multiple sclerosis; $\mathbf{N K}=$ natural killer; PBMC = peripheral blood mononuclear cells; $\mathbf{P C T}=$ procalcitonin; $\mathbf{R R}=$ relapsing-remitting; $\mathbf{T N F}=$ tumor necrosis factor; $\mathbf{T S H}=$ thyroid-stimulating hormone.

Alemtuzumab is a humanized immunoglobulin G1 monoclonal anti-CD52 antibody approved for active relapsing-remitting (RR) multiple sclerosis (MS) exerting its strong clinical efficacy by a specific pattern of depletion of CD52-positive immune cells followed by their repopulation. ${ }^{1-3}$ By binding to lymphocytes, alemtuzumab causes lysis and rapid depletion of these cells. ${ }^{4}$ As with most infused biological therapies, infusion-associated reactions (IAR) are frequently reported as adverse events for alemtuzumab treatment. In addition to cellular depletion, bystander effects including transient cell activation and triggered cytokine release are thought to cause alemtuzumab-specific IARs. ${ }^{5-7}$ Corticosteroid pretreatment and concomitant antihistaminergic and antipyretic drugs have been demonstrated to manage and to prevent IARs. ${ }^{3,8}$

From the Center of Clinical Neuroscience, University Hospital, Dresden, Germany.

Funding information and disclosures are provided at the end of the article. Go to Neurology.org/nn for full disclosure forms. The Article Processing Charge was paid by the authors.

This is an open access article distributed under the terms of the Creative Commons Attribution-NonCommercial-NoDerivatives License 4.0 (CC BY-NC-ND), which permits downloading and sharing the work provided it is properly cited. The work cannot be changed in any way or used commercially. 
Much immunologic and clinical data are available regarding long-term follow-up after treatment with alemtuzumab. ${ }^{9-11}$ However, limited information is available regarding acute immunologic and laboratory changes during the first alemtuzumab infusion week. In clinical trials, laboratory testing was only performed before and 1 month after alemtuzumab infusion. ${ }^{12,13}$ As IARs may mimic infections, acute changes in classical laboratory parameters during infusions by alemtuzumab may be of importance. Previous reports have evaluated the immediate impact on serum cytokine levels and acute phase proteins associated with IARs and transient clinical exacerbation in neurologic symptoms in initial off-label use of alemtuzumab in patients with MS. ${ }^{14,15}$ However, these reports are not based on the actual protocol for alemtuzumab infusion delivering 5 alemtuzumab infusions of 12 mg over 4 hours with prior 1,000 mg methylprednisolone infusions on days $1-3$, which were utilized in the phase 3 studies. ${ }^{8}$ Importantly, the preceding methylprednisolone infusions seem to mitigate a lot of the previously observed IARs. ${ }^{8}$ Nevertheless, the acute cellular and serologic effects of the first alemtuzumab infusion week have not been systemically analyzed. Specific infections, such as Listeria meningitis, seem to be more prevalent after alemtuzumab infu$\operatorname{sion}^{16}$; however, the exact effects on the acquired vs innate immune system directly after alemtuzumab application are unknown.

In this study, we evaluated 15 patients by monitoring patients' vital signs and routine blood analysis as well as utilizing sophisticated immunologic analyses including serum cytokine levels and changes in the proportion of immune cell subtypes including analysis of the acquired and innate immune system regarding cell frequency and function and clinical relevance in the first alemtuzumab infusion week.

METHODS Patients and study approval. In our study, we included 15 patients diagnosed with RRMS and active disease treated with alemtuzumab after critical review of clinical and MRI data and extensive discussion of available treatment options (table). The experiments were approved by the institutional review board of the University Hospital of Dresden. Patients gave their written informed consent.

Alemtuzumab infusion protocol and blood sampling. The alemtuzumab infusion protocol used in our MS center is based on the standardized infusion protocol described and utilized in
Comparison of Alemtuzumab and Rebif Efficacy in Multiple Sclerosis (CARE-MS) 1 and 2 clinical trials ${ }^{8,12,13}: 12 \mathrm{mg}$ alemtuzumab was given on 5 consecutive days IV over the course of 4 hours. On days 1-3, patients were pretreated with $1,000 \mathrm{mg} \mathrm{IV}$ methylprednisolone 1 hour before the alemtuzumab infusion. Patients received prophylactic antihistaminergic treatment including $5 \mathrm{mg}$ of oral desloratadine and $300 \mathrm{mg}$ ranitidine twice daily as well as $200 \mathrm{mg}$ of oral acyclovir twice daily beginning the evening before the first infusion. Before each alemtuzumab infusion, patients received $1 \mathrm{~g}$ IV paracetamol. Monitoring of vital signs including blood pressure, heart rate, and body temperature were performed each day before starting and hourly until 1 hour after the end of the alemtuzumab infusion. Blood samples were taken prior to methylprednisolone, after methylprednisolone, and after alemtuzumab administration on each of the 5 consecutive days (figure 1). To control for methylprednisolone effects, blood samples of 5 patients with RRMS, not receiving continuous immunomodulatory or immunosuppressive therapy, treated for 3 days with 1,000 mg IV methylprednisolone for acute relapse treatment served as controls.

Routine blood analysis. Standardized blood testing was performed for routine blood parameters at the Institute of Clinical Chemistry and Laboratory Medicine, University Hospital in Dresden, Germany. The institute complies with standards required by DIN-EN-ISO-15189:2014 for medical laboratories. Routine blood testing included complete blood cell count, liver enzymes, pancreatic-amylase, creatinine, sodium and potassium, thyroid-stimulating hormone (TSH), and acute-phase proteins including C-reactive protein (CRP), procalcitonin (PCT), lactate dehydrogenase (LDH), and complement components $\mathrm{c} 3$ and $c 4$.

Immune cell phenotyping by fluorescence-activated cell sorting. After blood collection, peripheral blood mononuclear cells (PBMC) were prepared by Ficoll-Hypaque (Biochrom, Berlin, Germany) density centrifugation. Subpopulations of $\mathrm{T}$ cells, $B$ cells, natural killer (NK) cells, and antigen-presenting cells (APC) were characterized by surface staining with fluorescencelabeled anti-CD3, anti-CD4, anti-CD8, anti-CD16, anti-CD14, anti-CD19, anti-CD56 (BD Biosciences, Heidelberg, Germany) or anti-BDCA2, and anti-slan (Miltenyi Biotec, Bergisch-Gladbach, Germany) according to the manufacturer's instructions. Negative controls included directly labeled or unlabeled isotype-matched irrelevant antibodies (BD Biosciences). Cells were evaluated on LSR-Fortessa (BD Biosciences).

Cytokine assay. During alemtuzumab infusions, serum samples were taken and directly frozen $\left(-80^{\circ} \mathrm{C}\right)$ after collection. Afterwards, serum was analyzed to obtain concentrations of interleukin (IL)-1 $\beta$, IL-2, IL-4, IL-5, IL-6, IL-9, IL-10, IL-12, IL-13, IL$17 \mathrm{~A}$, IL-22, interferon (IFN) $-\gamma$, and tumor necrosis factor (TNF) $-\alpha$ using a commercial multiplexed fluorescent beadbased immunoassay (eBioscience, Frankfurt, Germany) according to the manufacturer's instructions. To investigate the potential of PBMCs to release cytokines upon stimulation ex vivo after alemtuzumab infusions, freshly prepared PBMC were suspended in culture medium consisting of RPMI 1640 (Biochrom), 5\% human AB-serum (CC pro, Neustadt, Germany), $2 \mathrm{mM}$ L-glutamine, $100 \mathrm{U} / \mathrm{mL}$ penicillin, and 100 $\mu \mathrm{g} / \mathrm{mL}$ streptomycin (Biochrom), plated on round-bottomed $96-$ well plates at $2 \times 10^{5}$ cells per well, and maintained for 18 hours in cell culture. Cytostim (Miltenyi Biotec) was added for the last 4 hours to stimulate cytokine release in lymphocytes; lipopolysaccharide (LPS) (1 $\mu \mathrm{g} / \mathrm{mL}$; Sigma Aldrich) was included for the complete 18 hours to stimulate cytokine release in dendritic cells (DC) and monocytes. Unstimulated 


\begin{tabular}{|c|c|c|c|c|c|}
\hline Table & \multicolumn{5}{|c|}{ Characteristics of patients with multiple sclerosis included in the study } \\
\hline Patient no. & Sex & Age, y & EDSS & Pretreatment & Reason for changing treatment \\
\hline 1 & Female & 35 & 2.5 & Fingolimod & Planned pregnancy \\
\hline 2 & Female & 27 & 5.5 & Natalizumab & Highly active disease course \\
\hline 3 & Female & 25 & 1.5 & Treatment-naive & Highly active disease course \\
\hline 4 & Female & 48 & 2.0 & Natalizumab & High risk of $\mathrm{PML}, \mathrm{JCV}-\mathrm{Ab}+$ \\
\hline 5 & Male & 43 & 2.5 & Teriflunomide & Highly active disease course \\
\hline 6 & Female & 27 & 2.0 & Natalizumab & High risk of PML, JCV-Ab+ \\
\hline 7 & Female & 26 & 3.5 & Interferon- $\beta$ & Highly active disease course \\
\hline 8 & Female & 40 & 6.5 & Dimethyl fumarate & Highly active disease course \\
\hline 9 & Female & 30 & 2.0 & Fingolimod & Highly active disease course \\
\hline 10 & Female & 17 & 2.0 & Treatment-naive & Highly active disease course \\
\hline 11 & Male & 41 & 2.0 & Interferon- $\beta$ & Highly active disease course \\
\hline 12 & Female & 52 & 4.5 & Glatiramer acetate & Highly active disease course \\
\hline 13 & Female & 27 & 3.5 & Fingolimod & Highly active disease course \\
\hline 14 & Female & 31 & 3.5 & Interferon- $\beta$ & Highly active disease course \\
\hline 15 & Female & 31 & 4.0 & Fingolimod & Highly active disease course \\
\hline
\end{tabular}

Abbreviations: EDSS $=$ Expanded Disability Status Scale; JCV-Ab $=$ JC virus antibody; PML $=$ progressive multifocal leukoencephalopathy.

cells served as controls. Supernatants were collected and the concentrations of cytokines were determined using the commercial multiplexed fluorescent bead-based immunoassay as described above.

Statistical analysis. The Mann-Whitney test or Wilcoxon matched pairs test were used to assess the significance of the results. Values of $*_{p}<0.05,{ }^{* *} p<0.01$, and ${ }^{* * *} p<0.001$ were considered significant.

RESULTS Stable vital signs and mild adverse events. There were no relevant changes in blood pressure (figure $2 \mathrm{~A}$ ) or body temperature (figure $2 \mathrm{C}$ ) during the infusion procedure. All patients received antipyretics before each alemtuzumab infusion. Only one patient had febrile body temperature on day 4 within the last hours of alemtuzumab infusion. In all patients, heart rate was significantly increased within the last hours of alemtuzumab infusion on the first day, but remained within physiologic range during the following infusion days (figure 2B).

Adverse events were mostly mild during the infusion. Headache, fatigue, and nausea were the most common adverse events within the first days: $7 / 15$ patients on day $1,6 / 15$ patients on day 2, 3/15 patients on day $3,5 / 15$ patients on day 4, 4/15 patients on day 5. Exanthema was noticed in $5 / 15$ patients on day 4 and in $5 / 15$ patients on day 5 . After antihistamine and methylprednisolone administration, exanthema rapidly resolved in all treated patients. There were no severe adverse events.

Acute effects on blood cell count. After the first day of infusion, leukocyte counts increased in all patients but declined to normal during subsequent days (figure 3A.a). This observation could be primarily attributed to increased neutrophil counts (figure 3A.b), as the number of eosinophils and basophils dropped (figure 3, A.c and A.d). Absolute lymphocyte counts were decreased by the first day of infusion and remained low, close to the limit of detection (figure 3A.e). During infusion, monocytes and platelets decreased (figure 3, A.f and A.g). No changes were detectable for hemoglobin, hematocrit, or erythrocyte count (data not shown). One month after alemtuzumab infusion, all cell types recovered to physiologic values with the exception of the absolute lymphocyte count (figure 3, A.a-A.g).

Effects mediated by methylprednisolone treatment. Methylprednisolone-treated patients were analyzed in order to differentiate methylprednisolone and alemtuzumab effects. Patients with RRMS treated with methylprednisolone exhibited increases in leukocyte and neutrophil counts, whereas eosinophil and basophil counts were decreased (figure e-1, (a)A-D, at Neurology.org/nn). In contrast to the effects of alemtuzumab infusion, lymphocyte counts remained stable and monocytes even increased (figure e-1, (a)E-F) after methylprednisolone treatment. Of the investigated serologic parameters, only LDH levels slightly increased. All other parameters measured, including platelet count, liver enzymes, kidney function, TSH, and acute phase proteins, did not exhibit significant changes during methylprednisolone treatment (figure e-1, a-c). 
Figure 1 Time points of blood sampling

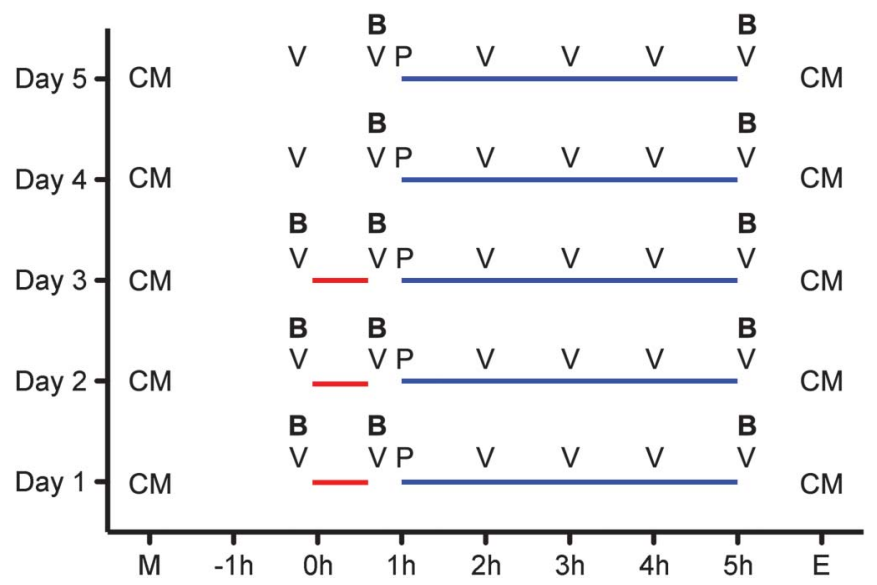

Alemtuzumab (blue line) was given on 5 consecutive days (day 1-day 5) within 4 hours. On days $1-3$, patients were pretreated with methylprednisolone (red line) 1 hour before alemtuzumab infusion. Blood samples (B) were taken before methylprednisolone, after methylprednisolone, and after alemtuzumab infusion each of the 5 consecutive days. Vital signs (V) were evaluated hourly. Paracetamol (P) was given before each alemtuzumab infusion. Patients received concomitant medication (CM) including antihistaminergic treatment and acyclovir in the morning $(\mathrm{M})$ and evening $(\mathrm{E})$ of each day.

Increase in liver enzymes. A significant increase, up to $2.5 \times$ the normal upper limit, was observed in alanine aminotransferase, $\gamma$-glutamyltransferase, and to a lesser extent, aspartate aminotransferase levels on days 3-5 (figure 3, B.a-B.c). Analysis of pancreatic amylase showed only a slight increase on day 5 as compared to baseline without reaching pathologic levels (figure 3B.d). Kidney function parameters including creatinine (figure 3B.e), glomerular filtration rate, as well as sodium and potassium levels (data not shown) remained stable within physiologic ranges. TSH dropped significantly on days 2-4, but returned to baseline values on day 5 (figure 3B.f). One month after alemtuzumab infusion, all measured parameters returned to physiologic values (figure 3, B.a-B.f, M1).

Increase in acute phase proteins unrelated to infection. After the first methylprednisolone and alemtuzumab infusion, CRP significantly increased up to the upper normal limit of $106 \mathrm{mg} / \mathrm{mL}$, declined in the following days, and peaked again on day 5 (figure 3C.a). Also, PCT markedly increased up to $55 \mathrm{ng} / \mathrm{mL}$ after alemtuzumab infusion (figure 3C.b). PCT and CRP returned to normal 1 month after alemtuzumab infusion (figure 3, C.a-C.b, M1). Furthermore, LDH significantly increased pathologically even after first alemtuzumab infusion and declined in the following days. Also, $\mathrm{LDH}$ returned to normal during 1 month follow-up (figure 3 C.c, M1). Complement activation was evaluated by complement c3 and c4 serum levels, which kept stable during the whole infusion cycle (figure 3, C.d-C.e).

Effects on selective immune cell subtypes. There were no significant effects directly after methylprednisolone infusion on $\mathrm{CD} 3+, \mathrm{CD} 4+, \mathrm{CD} 8+$, or $\mathrm{CD} 19+$ cell

Figure 2 Vital signs during alemtuzumab infusion cycle

A

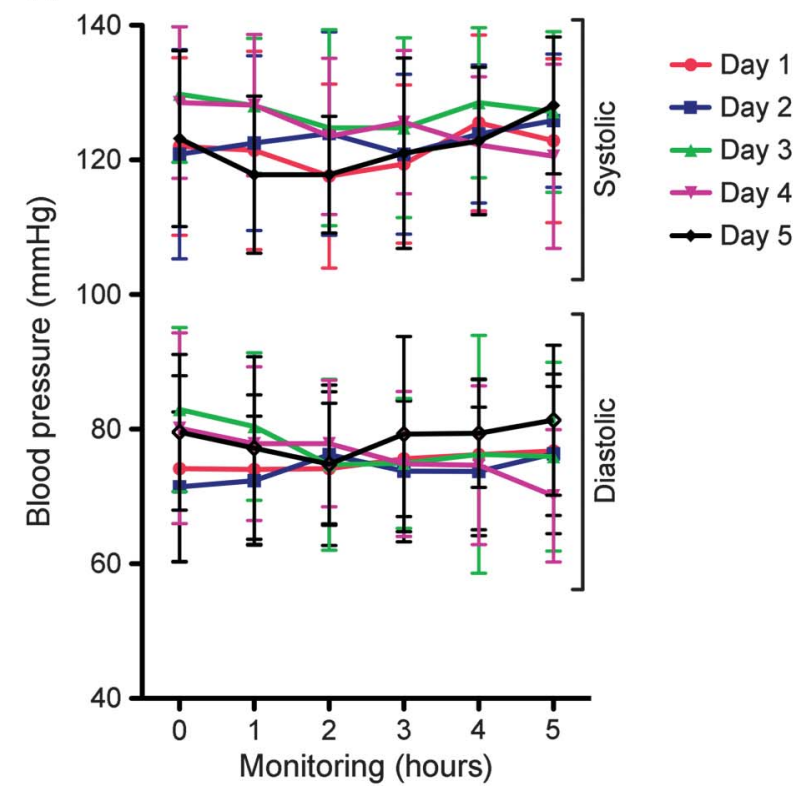

B
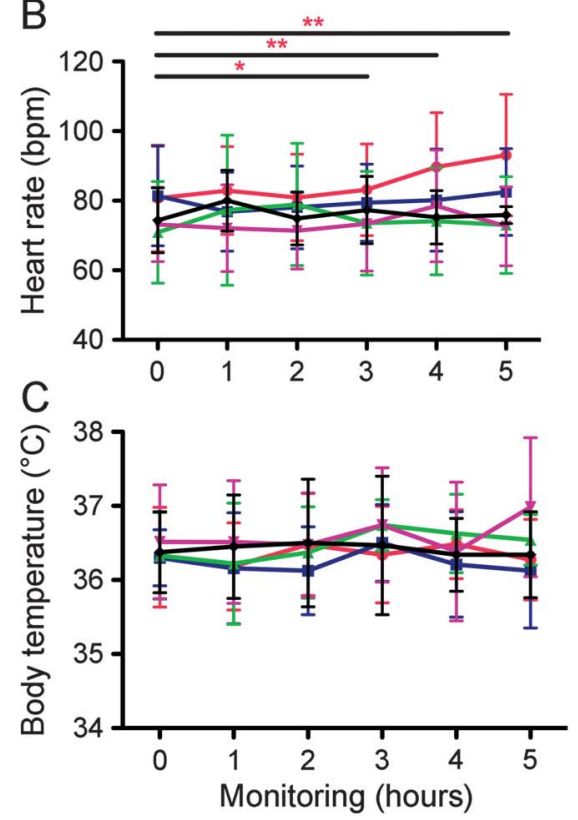

The progress of vital signs including blood pressure (A), heart rate (B), and body temperature (C) are depicted. Vital parameters were evaluated before methylprednisolone ( 0 hour), after methylprednisolone (1 hour), and each hour of alemtuzumab infusion (2-5 hours) each of the 5 days of infusion cycle (day 1-day 5). Values represent the mean \pm SD of each measured parameter from each of the 15 evaluated patients. Asterisks indicate a statistically significant difference on the indicated day $(* p<0.05, * * p<0.01)$. 
A.a

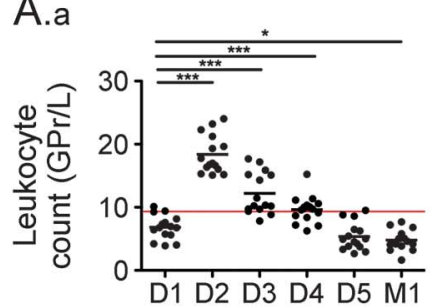

A.e

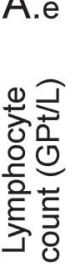

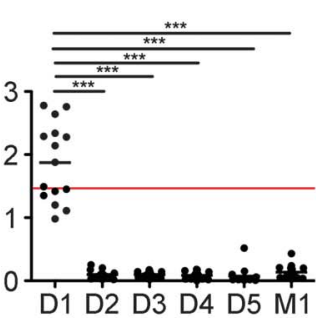

B.a

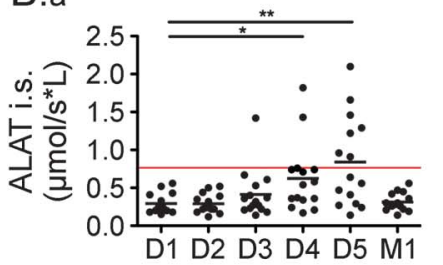

B.e

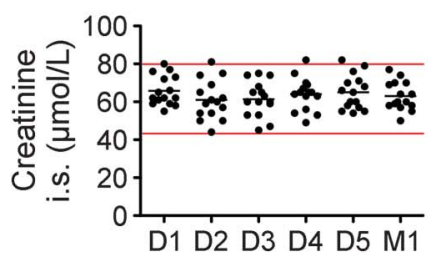

C.a

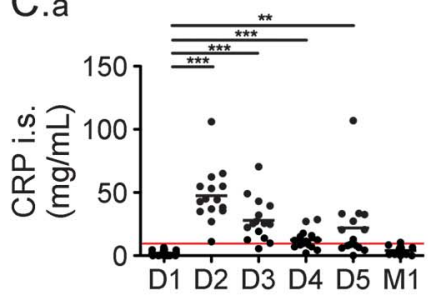

C.d

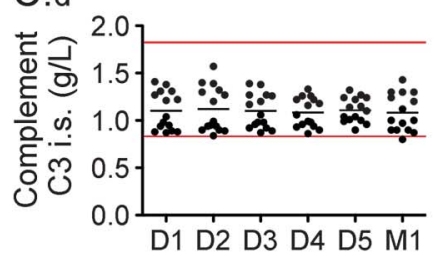

A.b

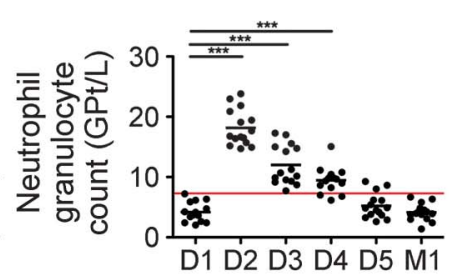

A.f

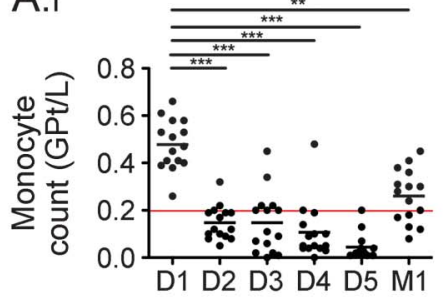

B.b

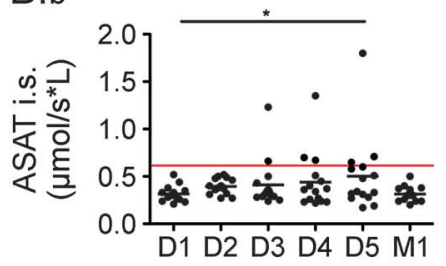

B.f

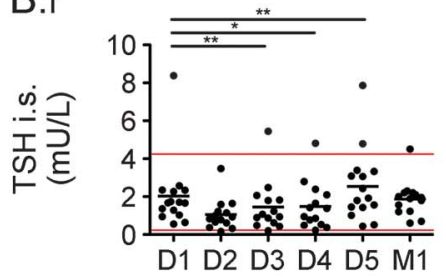

C.b

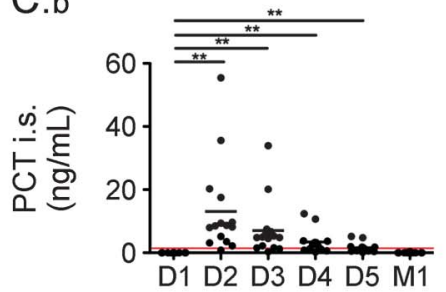

C.e

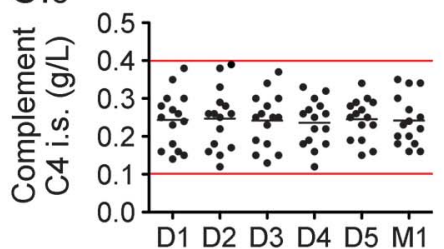

A.c

A.d

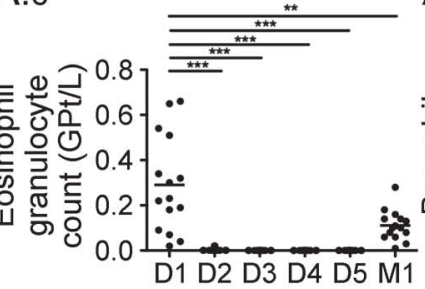

A.g
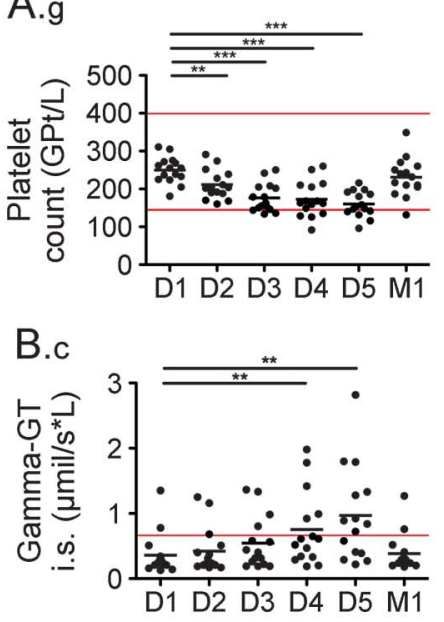

B.d

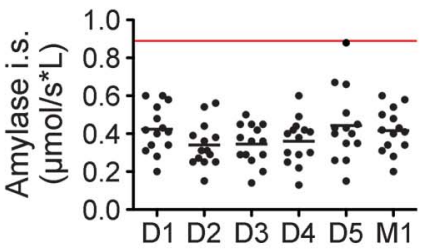

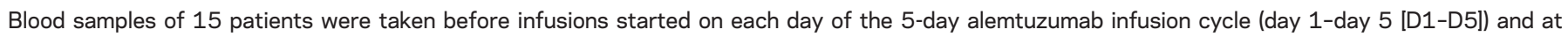

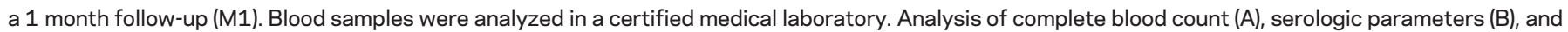

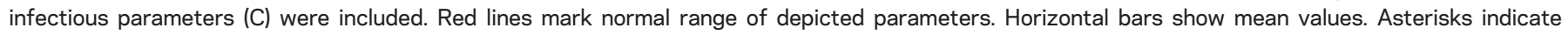

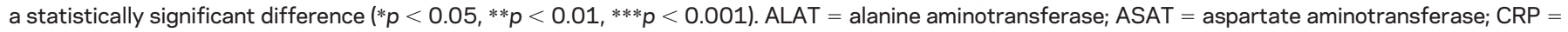
C-reactive protein; GT = glutamyltransferase; $\mathrm{LDH}=$ lactate dehydrogenase; PCT = procalcitonin; TSH = thyroid-stimulating hormone.

numbers. In contrast, alemtuzumab infusion led to a dramatic rapid drop in $\mathrm{CD} 3+, \mathrm{CD} 4+, \mathrm{CD} 8+$, and CD19+ lymphocytes (figure 4, A-D). Interestingly, partial recovery was observed overnight until the next day's infusion, which was again followed by a marked drop (figure 4, A-D, D2A). After day 3, $\mathrm{CD} 3+, \mathrm{CD} 4+, \mathrm{CD} 8+$, and $\mathrm{CD} 19+$ lymphocytes were present at very low frequencies in the blood 
Figure 4 Cell count of selective immune cell subpopulations during alemtuzumab

A

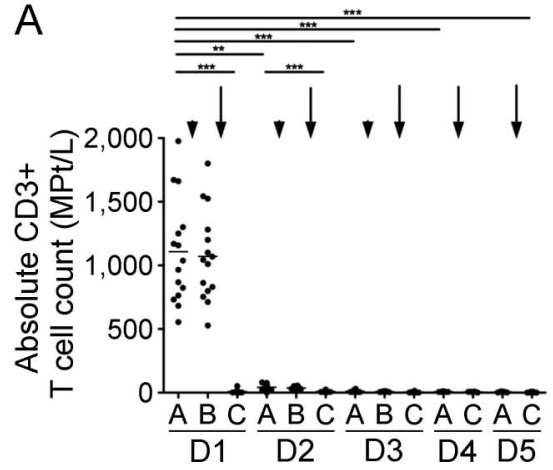

C
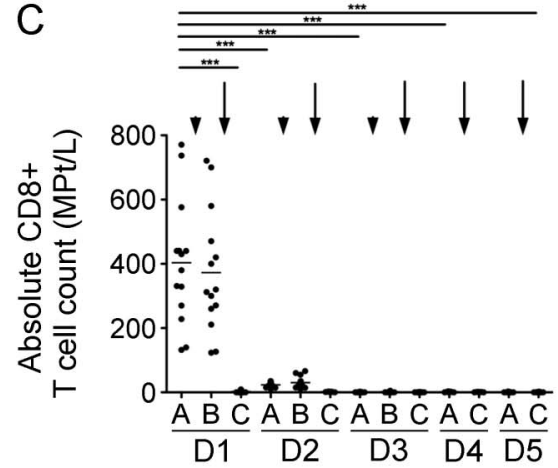

B
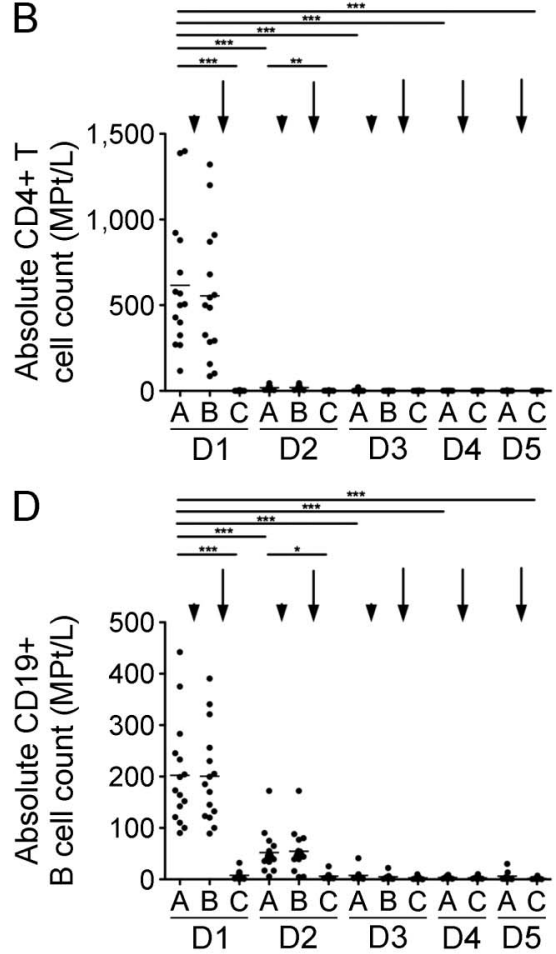

$E$

$\mathrm{F}$
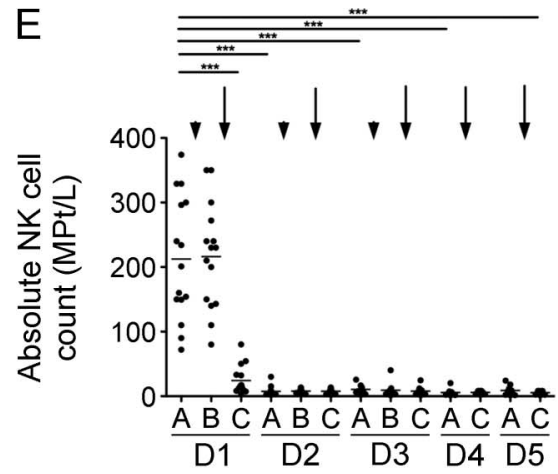

G
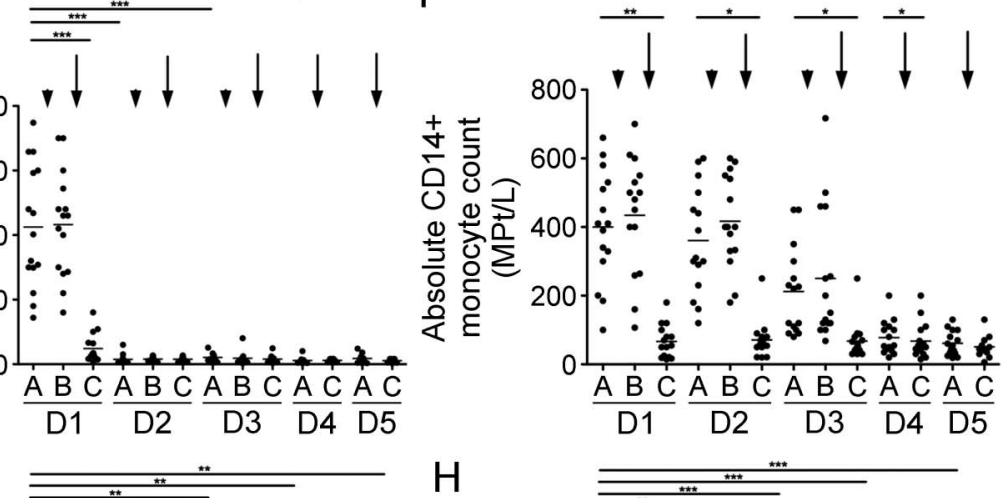

$\mathrm{H}$
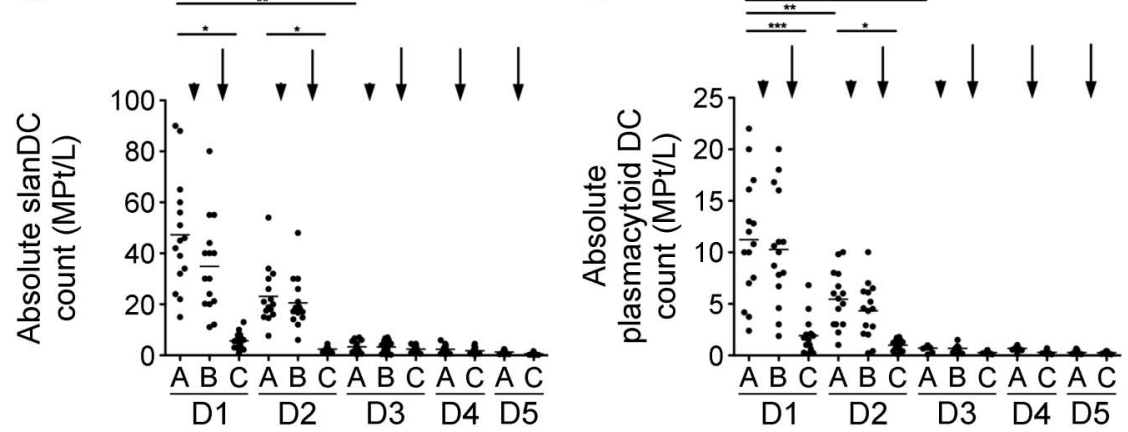

Absolute cell count of CD3 + T cells (A), CD4+ T cells (B), CD8 + T cells (C), CD19+ B cells (D), natural killer (NK) cells (E), CD14+ monocytes $(F)$, slanDC $(G)$, and plasmacytoid DC $(H)$ are depicted. Analysis was performed before infusions started (A), after methylprednisolone (B), and after alemtuzumab (C) on each of the 5 infusion days (day 1-day 5 [D1-D5]). Arrowhead indicates administration of methylprednisolone, arrow indicates administration of alemtuzumab. Horizontal bars show mean values. Asterisks indicate a statistically significant difference $(* p<0.05, * * p<0.01, * * * p<0.001)$.

(figure 4, A-D). In addition, NK cells dropped significantly after the first alemtuzumab infusion and remained stable at low levels (figure 4E). Each methylprednisolone infusion led to an increase in CD14+ monocytes, yet after alemtuzumab infusion monocyte counts dropped significantly. Monocytes partially recovered until the next infusion day (figure 4F). Alemtuzumab led to a significant drop in various 

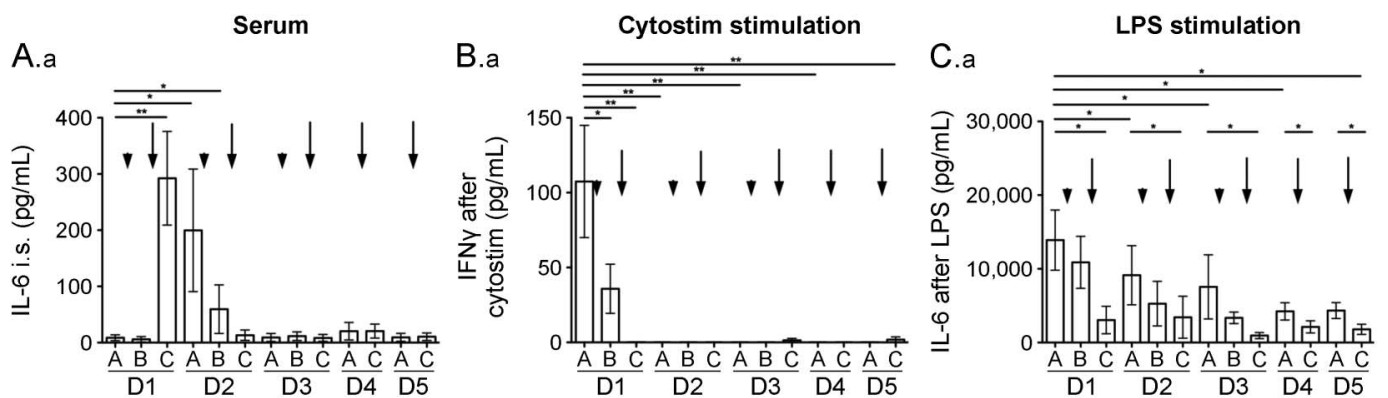

A.b

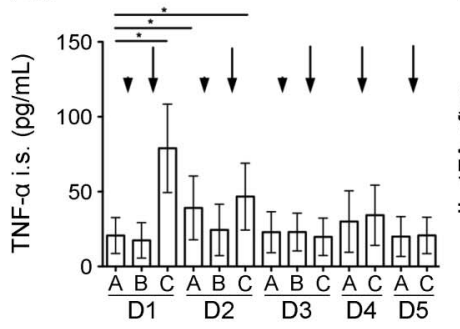

A.c

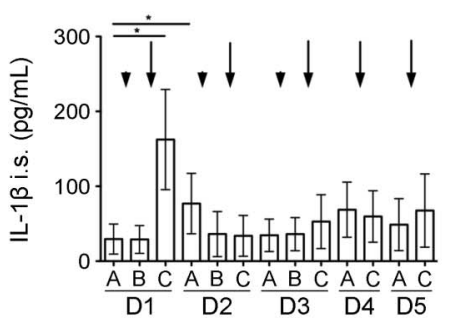

A.d

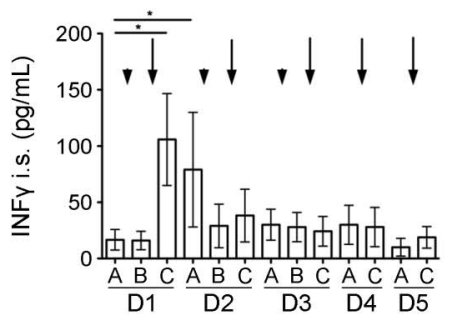

A.e

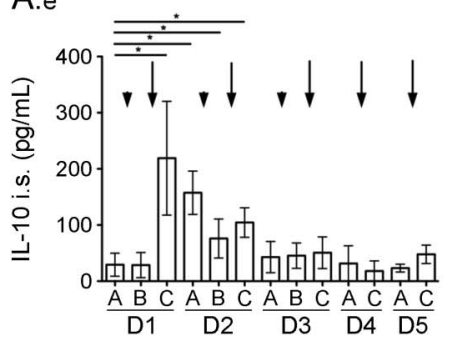

B.b

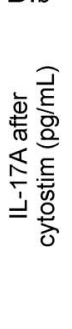

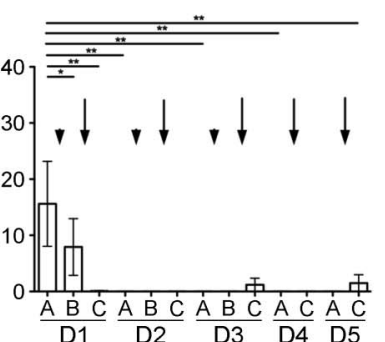

B.c

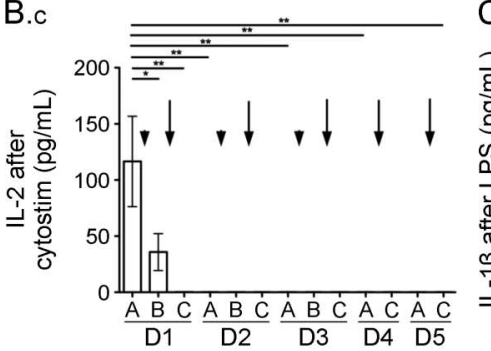

B.d
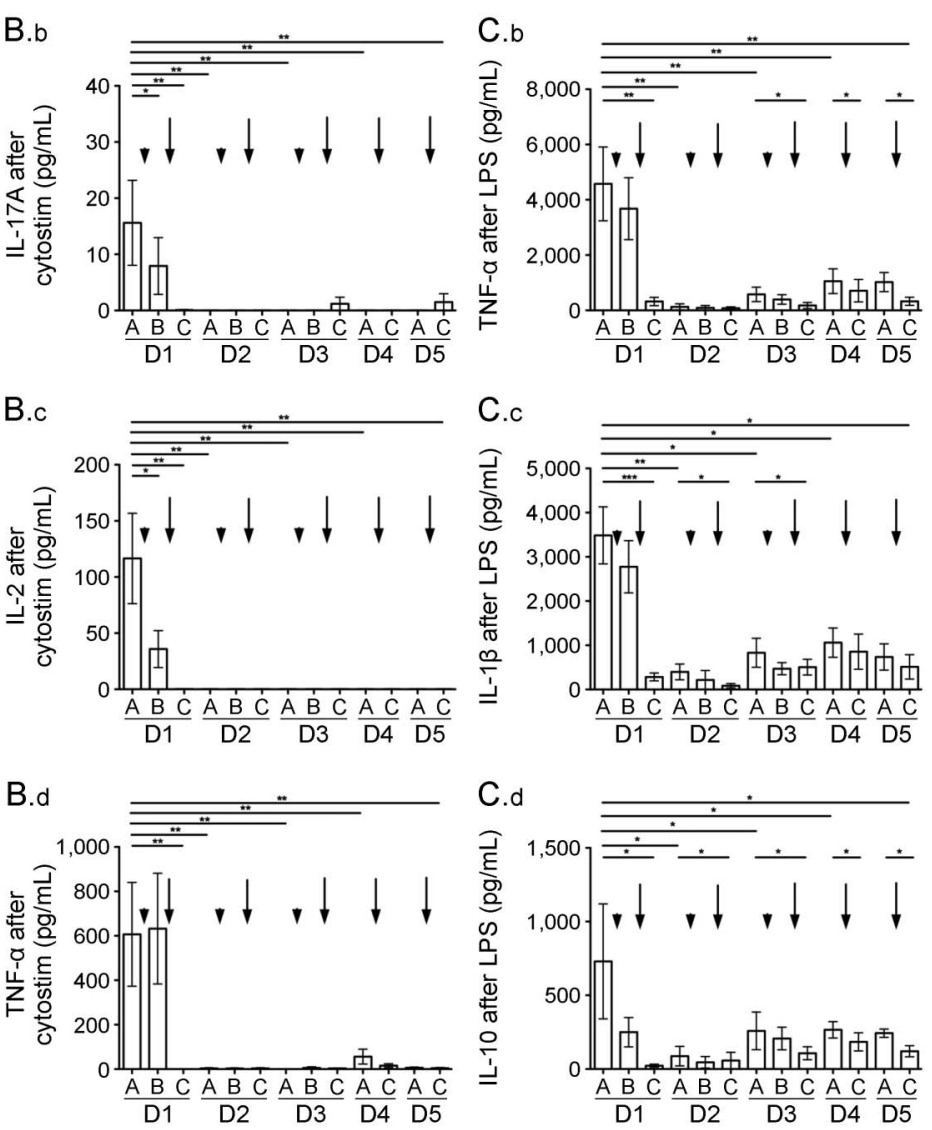

C.c

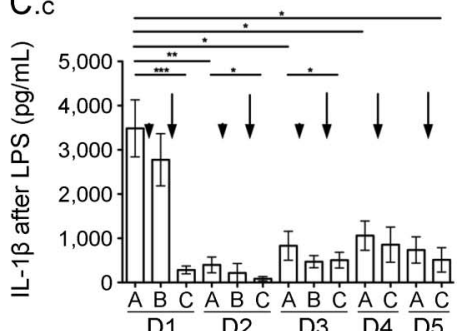

B.e

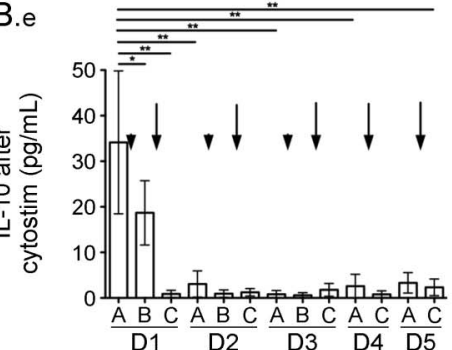

Cytokine serum levels of interleukin (IL)-6 (A.a), tumor necrosis factor (TNF)- $\alpha$ (A.b), IL-1 $\beta$ (A.c), interferon (IFN)- $\gamma$ (A.d), and IL-10 (A.e) during alemtuzumab infusion cycle are presented. Cytokine levels in cultured cell supernatants: IFN- $\gamma$ (B.a), IL-17A (B.b), IL-2 (B.c), TNF- $\alpha$ (B.d), and IL-10 (B.e) after ex vivo Cytostim stimulation and IL-6 (C.a), TNF- $\alpha$ (C.b), IL-1 $\beta$ (C.c), and IL-10 (C.d) after ex vivo LPS stimulation are depicted. Analysis was performed before infusions started (A), after methylprednisolone (B), and after alemtuzumab (C) each of the 5 infusion days (day 1-day 5 [D1-D5]). Arrowhead indicates administration of methylprednisolone, arrow indicates administration of alemtuzumab. Mean values \pm SEM are presented. Asterisks indicate a statistically significant difference $(* p<0.05, * * p<0.01, * * * p<0.001)$. 
DC populations including slan DCs and plasmacytoid DCs (figure 4, G and $\mathrm{H}$ ).

Increased serum cytokine levels and impaired immune cell activation after alemtuzumab infusion. Methylprednisolone had no impact on serum cytokine levels, but alemtuzumab infusion led to a significant increase in IL-6, IL-1 $\beta$, TNF- $\alpha$, IFN- $\gamma$, and IL-10 (figure 5, A.a-A.e, D1C). Each cytokine then decreased at unique rates over the next days of infusion (figure 5, A.a-A.e, D2-D5).

After stimulation of $\mathrm{T}$ cells with Cytostim, an increase in IFN- $\gamma$, IL-17A, IL-2, TNF- $\alpha$, and IL10 was documented in supernatants of stimulated cells collected prior to the first infusion (figure 5, B.a-B.e, D1A). After the first methylprednisolone infusion, the amount of IFN- $\gamma$, IL-17A, IL-2, and IL-10 produced after stimulation was slightly decreased (figure 5, B.a-B.e, D1B). After first alemtuzumab infusion, Cytostim-stimulated cells did not release any cytokines (figure 5, B.a-B.e) throughout the entire infusion cycle. However, stimulation of APCs with LPS led to a clear increases in IL-6, TNF- $\alpha$, IL-1 $\beta$, and IL-10 secretion (figure 5, C.a-C.d). This LPS-induced cytokine release was moderately inhibited after the first methylprednisolone infusion and significantly inhibited after the first alemtuzumab infusion (figure 5, C.a-C.d, D1B/C). Although the potential of APCs to produce cytokines on LPS challenge partially recovered on the following day, cytokine release remained diminished during the subsequent infusion days (figure 5, C.a-C.d). No cytokine expression was detectable in unstimulated cells (data not shown).

DISCUSSION For the first time, we have systematically analyzed the acute cellular and serologic effects of alemtuzumab during the first infusion week in 15 patients with active MS. Our data rely on the actual infusion procedure used during the phase 3 clinical trials, administering $12 \mathrm{mg}$ alemtuzumab on 5 consecutive days with preceding methylprednisolone infusion on days $1-3.8,12,13$ This is of importance as CD52-specific cell depletion is responsible for the high clinically efficacy as well as for the IARs common with cell-depleting monoclonal antibodies. In contrast to the previous off-label use of alemtuzumab, we present acute effects and their management during the use of a standardized infusion protocol, which is mandatory since alemtuzumab approval in clinical practice today. In $90 \%$ of treated patients who did not receive methylprednisolone pretreatment, alemtuzumab infusion causes an acute cytokine release syndrome consisting clinically of pyrexia, headache, malaise, and an urticarial rash and may be accompanied by a transient exacerbation of neurologic symptoms as described in premarketing reports. ${ }^{14,15}$ These symptoms can be managed by pretreatment with corticosteroids, antihistamines, and paracetamol $^{8}$ and are, in general, well-tolerated, which we observed in our 15 patients as well. Our patients complained about transient fatigue, headache, and nausea, but also mild to moderate exanthema, and presented with heart rate increases after the initial alemtuzumab infusion.

Cytokine release and lymphocyte activation could account for such IARs. ${ }^{5,14,15}$ Up until now, the detailed pathophysiology of this so-called cytokine release syndrome, especially observed after T-cell-targeted antibody treatments, is poorly understood. ${ }^{6,7,17}$ Induction of serum cytokines could be due to crosslinking of NK cells in addition to cell lysis. ${ }^{5}$ Although it is difficult to compare, our patients demonstrated lower levels of serum cytokines after initial alemtuzumab infusions as compared to other reports of cytokine levels that did not utilize our standardized infusion procedure and methylprednisolone pretreatment. ${ }^{14,17}$ These results are in line with fewer adverse events in our patient group and provide evidence that pretreatment and concomitant methylprednisolone treatment incorporated into the standard infusion protocol may attenuate cytokine release syndrome and help reduce IARs to enhance patient tolerance. ${ }^{8}$ One limitation of our study is that we did not measure histamine levels in our patients. As decrease of IAR symptoms was linked to the preventive or symptomatic use of antihistamine medications, pathophysiology of IARs seems to be histamine-modulated.

The alemtuzumab-mediated depletion of CD52expressing cells takes place rapidly after the initial alemtuzumab infusion, as seen in our 15 patients. Here, we report that alemtuzumab led to depletion within a fewminutes after infusion, which is known to be mediated via antibody-dependent cell-mediated cytolysis and complement-dependent cytolysis. Depletion within secondary lymphoid tissues appears trivial when extrapolating data from the human CD52 transgenic mouse. ${ }^{18}$ Differential depletion and repopulation could affect the number, proportion, and properties of different lymphocyte subsets, which in turn may lead to a rebalancing of the immune system responsible for the longterm effects of alemtuzumab. ${ }^{18-20}$ The rapid onset of alemtuzumab treatment effects can be explained by depletion of cells of the acquired and innate immune system, including the impairment in activation as well as cytokine release. Although there was a partial recovery on the second day, lymphocytes and monocytes were significantly decreased between infusion days 3 and 5 . According to our data, peripheral immune cell counts cannot be used to monitor quantity of alemtuzumab depletion during the infusion week as the window of blood cells is narrow and does not allow extrapolation on the effect in all immune compartments. ${ }^{21}$

Interestingly, cellular depletion and decreased activation with reduced cytokine release after stimulation 
could be demonstrated not only for lymphocytes, but also for cells of the innate immune system. We found a significant drop in cell frequency in blood and decreased cytokine secretion after ex vivo LPS stimulation for both monocytes and DCs, which play an important role in MS pathophysiology. ${ }^{22}$ However, these cells also provide the physical link between the innate and adaptive immune system, orchestrating the host defense against invading bacterial pathogens. ${ }^{23,24}$ The ability of alemtuzumab to deplete DCs has been previously described in chronic lymphocytic leukemia ${ }^{25}$ and may explain cases of Listeria meningitis linked to the initial alemtuzumab infusions. ${ }^{16}$ It is known that Listeria monocytogenes bacteria are phagocytosed by DCs via a serum-dependent mechanism. ${ }^{26}$ Listeria meningitis infection related to alemtuzumab infusion may be evoked by a preexisting, clinically silent, and CD8+ T-cell-controlled infection due to cellular depletion and activation blockade of adaptive and innate immune cells. These findings highlight the relevance of certain infections, which could be promoted by the depletion and blockade of innate immune subsets, of which clinicians should be aware within the first days after initial alemtuzumab infusion.

As infections can be facilitated by cellular depletion of the adaptive and innate immune system together and IAR symptoms like fever, headache, and fatigue may mimic infectious signs, routine blood analysis is usually a suitable tool to identify infectious conditions. While CRP has been reported as a useful marker of viral and bacterial infection, synthesis of CRP may be reduced during liver failure or after steroid treatment. ${ }^{27,28} \mathrm{PCT}$ is produced in response to endotoxin and to cytokines involved in the sepsis cascade. ${ }^{29}$ In immunocompetent and neutropenic septic patients, the prognostic value of PCT has been shown to be higher than that of CRP. ${ }^{30}$ In our patients, alemtuzumab infusion resulted in a marked increase in both acute phase proteins, especially on the first alemtuzumab infusion day. Some patients presented with PCT and CRP levels typical for acute septic disease, ${ }^{31}$ although all patients felt well and no infectious condition could be identified. Increases in CRP and PCT not linked to infection have been previously reported for other monoclonal antibody treatments, ${ }^{32,33}$ but methylprednisolone was not used as in the alemtuzumab protocol. Again, this phenomenon may be explained by lymphocyte destruction and cytokine-mediated effects. ${ }^{14,24,25}$ A profound stimulatory effect of TNF- $\alpha$ on PCT is sometimes observed. $^{34}$ After administration of TNF- $\alpha$, PCT reached half-maximal concentrations within $8-12$ hours earlier than CRP. ${ }^{34}$ Hepatotoxicity could also be considered as a potential factor of CRP increase as we were able to demonstrate a distinct increase in liver enzymes as well. Our systematic analysis indicates that clinicians should be aware of the characteristic increase in CRP and PCT during the standard alemtuzumab infusion procedure. As leukocytes are markedly affected by infusions of methylprednisolone and alemtuzumab as well, these parameters are also not useful as a screen for potential infections, which are more frequent in cell-depleted patients. Thus, the need for additional analysis in such patients as seen in 2 cases of Listeria meningitis presenting with increased cell counts in CSF during the first alemtuzumab infusion week becomes apparent. ${ }^{16}$ Acutephase proteins such as CRP and PCT are of limited value as diagnostic markers of severe bacterial infections during alemtuzumab treatment. A combination of a detailed clinical assessment and careful interpretation of collateral biochemical and microbiologic tests probably remains the optimal approach to these patients. Daily standard blood testing does not seem to be helpful during alemtuzumab infusion weeks as many cellular and serologic artifacts are observed that are not conducive to clinical interpretation. We suggest using blood tests only if indicated and knowing the characteristic alemtuzumab effects.

While kidney function was not affected by alemtuzumab treatment, liver enzymes demonstrated an increase during the last days of the alemtuzumab infusion protocol. We assume a direct alemtuzumab effect, although a delayed impact of methylprednisolone cannot be excluded. We found that liver enzymes peaked at less than $3 \times$ upper normal limit and returned to normal in 30 days. Other clinically nonsignificant laboratory findings include a transient decrease of platelet count, not indicating or comparable with immune thrombocytopenic purpura, which takes place months to years after alemtuzumab infusion. ${ }^{35,36}$ Drug-induced thrombocytopenia has been described for several drugs with resolution after discontinuation. ${ }^{37} \mathrm{~A}$ comparable phenomenon was observed for TSH modulation, not associated with autoimmune thyroid disease occurring months to years later. ${ }^{38} \mathrm{TSH}$, reflecting thyroid function, is known to be modulated in various clinical conditions. ${ }^{39,40}$

We present rapid cellular depletion and impairment of activation of different adaptive and innate immune cell subtypes accompanied by marked serum cytokine increases within the first alemtuzumab infusion week. Standardized infusion procedures including pretreatment management can attenuate immunologic and clinical effects of alemtuzumab. Nevertheless, these acute alemtuzumabmediated effects are assumed to lead to several effects observable by standard blood testing, such as noninfectious increase in leukocyte count, CRP, and PCT, which are of particular importance in identification and monitoring of infectious conditions. Furthermore, transient elevation of liver enzymes, 
thrombocytopenia, and TSH modulation are demonstrated. Thus, we recommend clinicians be aware of clinical symptoms and vital data to initiate supportive analysis rather than standard laboratory testing within the first alemtuzumab treatment week.

\section{AUTHOR CONTRIBUTIONS}

Study concept and design: K. Thomas and T. Ziemssen. Acquisition of data: K. Thomas, J. Eisele, F.A. Rodriguez-Leal, U. Hainke. Analysis and interpretation of data: K. Thomas, J. Eisele, T. Ziemssen. Drafting of the manuscript: K. Thomas and J. Eisele. Critical revision of the manuscript for important intellectual content: F.A. Rodriguez-Leal, U. Hainke, T. Ziemssen. Statistical analysis: K. Thomas. Administrative, technical, and material support: K. Thomas and T. Ziemssen. Study supervision: T. Ziemssen.

\section{ACKNOWLEDGMENT}

The authors thank M. Marggraf and N. Kretschmann for technical assistance. This manuscript was edited by native English-Speaking experts from BioMed Proofreading LLC.

\section{STUDY FUNDING}

No targeted funding reported.

\section{DISCLOSURES}

K. Thomas received speaker honoraria from Novartis, Bayer, and Biogen Idec. J. Eisele and F.A. Rodriguez-Leal report no disclosures. U. Hainke received travel support from Novartis and Biogen Idec. T. Ziemssen is on the scientific advisory board for Bayer Healthcare, Biogen Idec, Genzyme, MSD, GDSK, Novartis, Teva, Sanofi, and Almirall; is section editor for BMC Neurology; and received research support from Bayer Healthcare, Biogen Idec, Genzyme, Novartis, Teva, and Sanofi. Go to Neurology.org/nn for full disclosure forms.

Received February 1, 2016. Accepted in final form February 24, 2016.

\section{REFERENCES}

1. Fox EJ. Alemtuzumab in the treatment of relapsingremitting multiple sclerosis. Expert Rev Neurother 2010; 10:1789-1797.

2. Hartung HP, Aktas O, Boyko AN. Alemtuzumab: a new therapy for active relapsing-remitting multiple sclerosis. Mult Scler 2015;21:22-34

3. Ruck T, Bittner S, Wiendl H, Meuth SG. Alemtuzumab in multiple sclerosis: mechanism of action and beyond. Int J Mol Sci 2015;16:16414-16439.

4. Minagar A, Alexander JS, Sahraian MA, Zivadinov R. Alemtuzumab and multiple sclerosis: therapeutic application. Expert Opin Biol Ther 2010;10:421-429.

5. Wing MG, Moreau T, Greenwood J, et al. Mechanism of first-dose cytokine-release syndrome by CAMPATH 1-H: involvement of CD16 (FcgammaRIII) and CD11a/CD18 (LFA-1) on NK cells. J Clin Invest 1996;98:2819-2826.

6. Lee DW, Gardner R, Porter DL, et al. Current concepts in the diagnosis and management of cytokine release syndrome. Blood 2014;124:188-195.

7. Maggi E, Vultaggio A, Matucci A. Acute infusion reactions induced by monoclonal antibody therapy. Expert Rev Clin Immunol 2011;7:55-63.

8. Caon C, Namey M, Meyer C, et al. Prevention and management of infusion-associated reactions in the comparison of alemtuzumab and Rebif $\left({ }^{\circledR}\right)$ efficacy in multiple sclerosis (CARE-MS) program. Int J MS Care 2015;17:191-198.

9. Tuohy O, Costelloe L, Hill-Cawthorne G, et al. Alemtuzumab treatment of multiple sclerosis: long-term safety and efficacy. J Neurol Neurosurg Psychiatry 2015;86: 208-215.

10. Kousin-Ezewu O, Azzopardi L, Parker RA, et al. Accelerated lymphocyte recovery after alemtuzumab does not predict multiple sclerosis activity. Neurology 2014;82:21582164.

11. Hill-Cawthorne GA, Button T, Tuohy O, et al. Long term lymphocyte reconstitution after alemtuzumab treatment of multiple sclerosis. J Neurol Neurosurg Psychiatry 2012; 83:298-304.

12. Cohen JA, Coles AJ, Arnold DL, et al. Alemtuzumab versus interferon beta 1a as first-line treatment for patients with relapsing-remitting multiple sclerosis: a randomised controlled phase 3 trial. Lancet 2012;380:1819-1828.

13. Coles AJ, Twyman CL, Arnold DL, et al. Alemtuzumab for patients with relapsing multiple sclerosis after diseasemodifying therapy: a randomised controlled phase 3 trial. Lancet 2012;380:1829-1839.

14. Moreau T, Coles A, Wing M, et al. Transient increase in symptoms associated with cytokine release in patients with multiple sclerosis. Brain 1996;119:225-237.

15. Moreau T, Coles A, Wing M, et al. CAMPATH-IH in multiple sclerosis. Mult Scler 1996;1:357-365.

16. Rau D, Lang M, Harth A, et al. Listeria meningitis complicating alemtuzumab treatment in multiple sclerosis: report of two cases. Int J Mol Sci 2015;16: 14669-14676.

17. Pruzanski W, Urowitz MB, Grouix B, Vadas P. Induction of TNF-alpha and proinflammatory secretory phospholipase A2 by intravenous administration of CAMPATH-1H in patients with rheumatoid arthritis. J Rheumatol 1995; 22:1816-1819.

18. Hu Y, Turner MJ, Shields J, et al. Investigation of the mechanism of action of alemtuzumab in a human CD52 transgenic mouse model. Immunology 2009; 128:260-270.

19. Lowenstein H, Shah A, Chant A, Khan A. Different mechanisms of Campath-1H-mediated depletion for CD4 and CD8 T cells in peripheral blood. Transpl Int 2006;19:927-936.

20. Zhang X, Tao Y, Chopra M, et al. Differential reconstitution of $\mathrm{T}$ cell subsets following immunodepleting treatment with alemtuzumab (anti-CD52 monoclonal antibody) in patients with relapsing-remitting multiple sclerosis. J Immunol 2013;191:5867-5874.

21. Blum KS, Pabst R. Lymphocyte numbers and subsets in the human blood. Do they mirror the situation in all organs? Immunol Lett 2007;108:45-51.

22. Thomas K, Dietze K, Wehner R, et al. Accumulation and therapeutic modulation of 6-sulfo $\operatorname{LacNAc}(+)$ dendritic cells in multiple sclerosis. Neurol Neuroimmunol Neuroinflamm 2014;1:e33. doi: 10.1212/NXI.0000000000000033.

23. Swiecki M, Colonna M. The multifaceted biology of plasmacytoid dendritic cells. Nat Rev Immunol 2015; 15:471-485.

24. Steinman RM, Banchereau J. Taking dendritic cells into medicine. Nature 2007;449:419-426.

25. Auffermann-Gretzinger S, Eger L, Schetelig J, Bornhauser M, Heidenreich F, Ehninger G. Alemtuzumab depletes dendritic cells more effectively in blood than in skin: a pilot study in patients with chronic lymphocytic leukemia. Transplantation 2007;83:1268-1272.

26. Kolb-Maurer A, Kurzai O, Goebel W, Frosch M. The role of human dendritic cells in meningococcal and listerial meningitis. Int J Med Microbiol 2003;293:241-249. 
27. Aufweber E, Jorup-Ronstrom C, Edner A, Hansson LO. C-reactive protein sufficient as screening test in bacterial vs. viral infections. J Infect 1991;23:216-220.

28. Massaro KS, Costa SF, Leone C, Chamone DA. Procalcitonin (PCT) and C-reactive protein (CRP) as severe systemic infection markers in febrile neutropenic adults. BMC Infect Dis 2007;7:137.

29. Muller B, White JC, Nylen ES, Snider RH, Becker KL, Habener JF. Ubiquitous expression of the calcitonin-i gene in multiple tissues in response to sepsis. J Clin Endocrinol Metab 2001;86:396-404.

30. Persson L, Soderquist B, Engervall P, Vikerfors T, Hansson LO, Tidefelt U. Assessment of systemic inflammation markers to differentiate a stable from a deteriorating clinical course in patients with febrile neutropenia. Eur J Haematol 2005;74:297-303.

31. Christ-Crain M, Muller B. Procalcitonin in bacterial infections: hype, hope, more or less? Swiss Med Wkly 2005; 135:451-460.

32. Dornbusch HJ, Strenger V, Sovinz P, et al. Non-infectious causes of elevated procalcitonin and C-reactive protein serum levels in pediatric patients with hematologic and oncologic disorders. Support Care Cancer 2008;16: 1035-1040.

33. Dornbusch HJ, Strenger V, Kerbl R, et al. Procalcitonin and C-reactive protein do not discriminate between febrile reaction to anti-T-lymphocyte antibodies and Gramnegative sepsis. Bone Marrow Transplant 2003;32:941-945.

34. Oberhoffer M, Stonans I, Russwurm S, et al. Procalcitonin expression in human peripheral blood mononuclear cells and its modulation by lipopolysaccharides and sepsisrelated cytokines in vitro. J Lab Clin Med 1999;134:49-55.

35. Cossburn M, Pace AA, Jones J, et al. Autoimmune disease after alemtuzumab treatment for multiple sclerosis in a multicenter cohort. Neurology 2011;77:573-579.

36. Cuker A, Coles AJ, Sullivan H, et al. A distinctive form of immune thrombocytopenia in a phase 2 study of alemtuzumab for the treatment of relapsing-remitting multiple sclerosis. Blood 2011;118:6299-6305.

37. George JN, Raskob GE, Shah SR, et al. Drug-induced thrombocytopenia: a systematic review of published case reports. Ann Intern Med 1998;129:886-890.

38. Coles AJ, Wing M, Smith S, et al. Pulsed monoclonal antibody treatment and autoimmune thyroid disease in multiple sclerosis. Lancet 1999;354:1691-1695.

39. Franklyn JA, Ramsden DB, Sheppard MC. The influence of age and sex on tests of thyroid function. Ann Clin Biochem 1985;22:502-505.

40. Andersen S, Pedersen KM, Bruun NH, Laurberg P. Narrow individual variations in serum $\mathrm{T}(4)$ and $\mathrm{T}(3)$ in normal subjects: a clue to the understanding of subclinical thyroid disease. J Clin Endocrinol Metab 2002;87:1068-1072. 


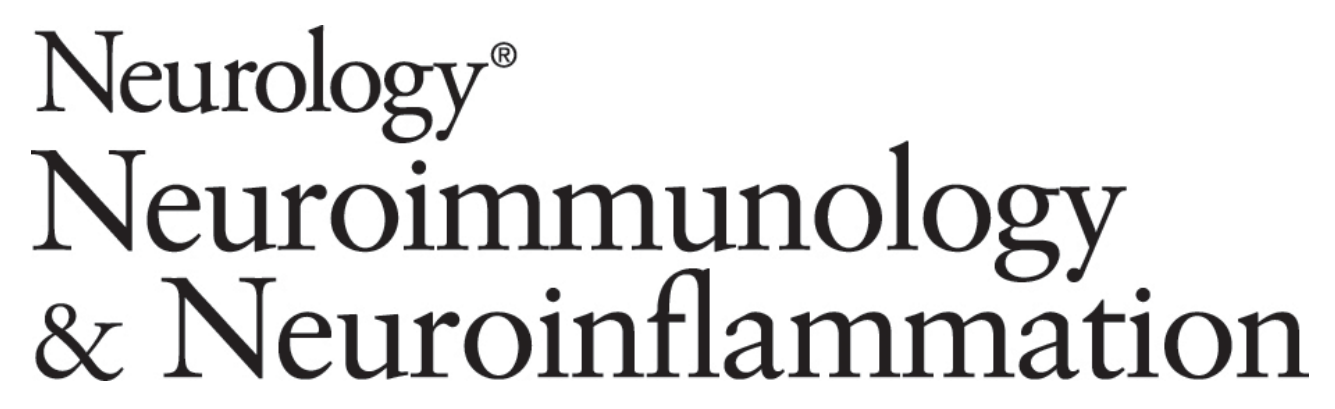

Acute effects of alemtuzumab infusion in patients with active relapsing-remitting MS Katja Thomas, Judith Eisele, Francisco Alejandro Rodriguez-Leal, et al.

Neurol Neuroimmunol Neuroinflamm 2016;3;

DOI 10.1212/NXI.0000000000000228

This information is current as of April 29, 2016

Neurol Neuroimmunol Neuroinflamm is an official journal of the American Academy of Neurology.

Published since April 2014, it is an open-access, online-only, continuous publication journal. Copyright $\odot$ 2016 American Academy of Neurology. All rights reserved. Online ISSN: 2332-7812.

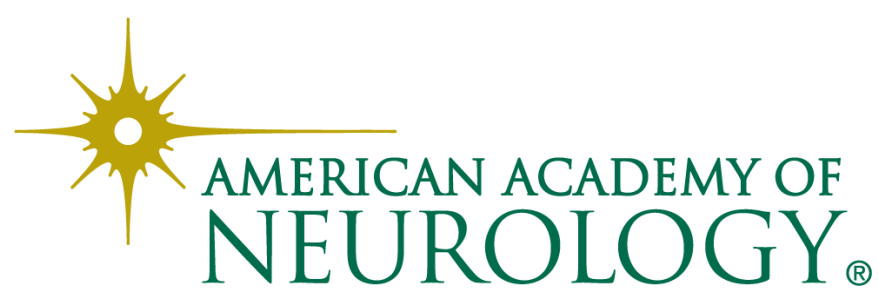




\section{Updated Information \& Services}

\section{Supplementary Material}

\section{References}

Citations

Subspecialty Collections

Permissions \& Licensing

Reprints including high resolution figures, can be found at: http://nn.neurology.org/content/3/3/e228.full.html

Supplementary material can be found at: http://nn.neurology.org/content/suppl/2016/05/02/3.3.e228.DC1

This article cites 40 articles, 5 of which you can access for free at: http://nn.neurology.org/content/3/3/e228.full.html\#\#ref-list-1

This article has been cited by 5 HighWire-hosted articles: http://nn.neurology.org/content/3/3/e228.full.html\#\#otherarticles

This article, along with others on similar topics, appears in the following collection(s):

\section{All Immunology}

http://nn.neurology.org//cgi/collection/all_immunology

Diagnostic test assessment

http://nn.neurology.org//cgi/collection/diagnostic_test_assessment_ Harm/ risk (analysis)

http://nn.neurology.org//cgi/collection/harm_risk_analysis

Multiple sclerosis

http://nn.neurology.org//cgi/collection/multiple_sclerosis

Information about reproducing this article in parts (figures,tables) or in its entirety can be found online at:

http://nn.neurology.org/misc/about.xhtml\#permissions

Information about ordering reprints can be found online: http://nn.neurology.org/misc/addir.xhtml\#reprintsus

Neurol Neuroimmunol Neuroinflamm is an official journal of the American Academy of Neurology.

Published since April 2014, it is an open-access, online-only, continuous publication journal. Copyright $\odot$ 2016 American Academy of Neurology. All rights reserved. Online ISSN: 2332-7812.

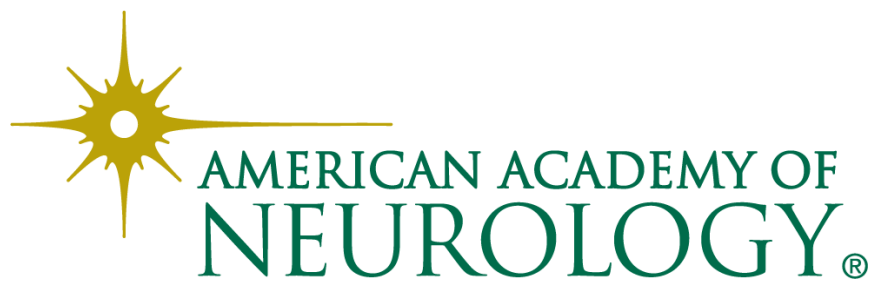

